\title{
Laser-based acceleration for nuclear physics experiments at ELI-NP
}

\author{
O. Tesileanu, Th. Asavei, I. Dancus, S. Gales, F. Negoita, \\ I.C.E. Turcu, D. Ursescu and N.V. Zamfir
}

\section{Extreme Light Infrastructure - Nuclear Physics, "Horia Hulubei" National R\&D Institute for Physics and Nuclear Engineering (IFIN-HH), 30 Reactorului street, 077125 Măgurele, Romania}

\begin{abstract}
As part of the Extreme Light pan-European research infrastructure, Extreme Light Infrastructure - Nuclear Physics (ELI-NP) in Romania will focus on topics in Nuclear Physics, fundamental Physics and applications, based on very intense photon beams. Laser-based acceleration of electrons, protons and heavy ions is a prerequisite for a multitude of laser-driven nuclear physics experiments already proposed by the international research community. A total of six outputs of the dualamplification chain laser system, two of $100 \mathrm{TW}$, two of $1 \mathrm{PW}$ and two of $10 \mathrm{PW}$ will be employed in 5 experimental areas, with the possibility to use long and short focal lengths, gas and solid targets, reaching the whole range of laser acceleration processes. We describe the main techniques and expectations regarding the acceleration of electrons, protons and heavy nuclei at ELI-NP, and some physics cases for which these techniques play an important role in the experiments.
\end{abstract}

\section{Introduction}

Extreme Light Infrastructure (ELI) is a distributed European research infrastructure dedicated to the generation and use in various experiments of extremely intense photon beams. The project initiated a decade ago with 
the idea of reaching $100 \mathrm{PW}$ laser powers by the coherent combination of several beams with powers of tens of PW, gained recognition by being adoped in the ESFRI (European Strategy Forum for Research Infrastructures) list of high-priority research projects in Europe in 2006 and then it was defined by a Preparatory Phase (ELI-PP, 2007-2010) funded by FP7.

ELI-PP reached the conclusion that, given the dimensions and extremely large scientific scope of the newly proposed research infrastructure, the best implementation strategy would be to adopt a distributed model, with the first three pillars to be hosted in three European countries: the Czech Republic, Hungary and Romania. These will focus on laser development up to the 10PW level and the related scientific applications. A fourth pillar would be decided later on, aiming, based on the experience of the first three pillars, to reach the hundreds of Petawatts power level.

The Romanian pillar of ELI is ELI-NP, devoted to experiments in laser science, nuclear physics and related areas, including applications, employing two 10PW laser beams and an extremely intense gamma photons beam. This unique combination of beams that are at their turn unique worldwide assures for ELI-NP the possibility to tackle areas at the frontiers of presentday knowledge and advance science in many areas, from the fundamental studies to society-relevant developments. The block diagram of ELI-NP presented in Fig.1 shows the large research equipment and the experimental areas with the corresponding science topics.

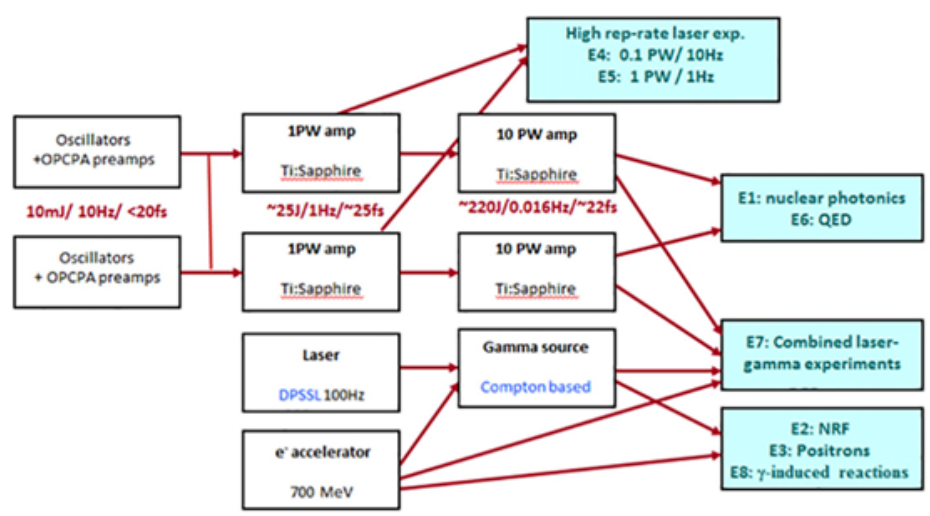

Figure 1: Block diagram of the ELI-NP infrastructure [16].

Technical Design Reports (TDR) for the various types of experiments envisaged at ELI-NP were developed and then reviewed by an international 
scientific advisory board. At the time of writing the experimental setups are entering the implementation phase.

\section{The ELI-NP high-power laser system (HPLS)}

The ELI-NP HPLS is a complex ultra-short pulse laser system, having two parallel amplification chains up to 10PW power, with intermediate outputs at $100 \mathrm{TW}$ and $1 \mathrm{PW}$ levels. Starting from the same front-end, pulses are stretched and amplified to a final energy in the range of $220 \mathrm{~J}$, and then compressed to pulse lengths of $22 \mathrm{fs}$.

HPLS will be constructed by an association between Thales Optronique SA France and Thales Romania. It consists of a chirped pulse amplification system at $\sim 820 \mathrm{~nm}$ central wavelength, with a dual front-end architecture (to minimize down-time for the laser facility). Each of the two parallel chains includes Ti:Sapphire amplifiers. The repetition rate for the two 10PW outputs is one shot per minute. The general layout of the HPLS laser system is similar with the one under development in France for CILEX-APOLLON facility [1].

The additional outputs at $0.1 \mathrm{PW}$ and $1 \mathrm{PW}$ along the two amplification chains, will be installed with the corresponding optical compressors. The pulse repetition rates for these outputs are $10 \mathrm{~Hz}$ and $1 \mathrm{~Hz}$, respectively. The pre-pulse temporal contrast for all the outputs is specified to be better than $10^{12}: 1$ both at picosecond and nanosecond delays and the requested Strehl ratio is 0.9 . The intensity to be attained is $\sim 10^{23} \mathrm{~W} / \mathrm{cm}^{2}$, securing a properly defined laser pulse in both space and time for interaction with thin targets. The energy stability is expected to reach $5 \%$ RMS and the pointing stability shall be less than 5 microrad RMS for both axes. In order to create the conditions to attain the pointing stability specifications the building has a vibration-damped floor across the entire laser and experiments areas.

\section{Laser-based acceleration of particles}

The acceleration of particles with the help of lasers is one of the research areas of great interest nowadays due to a multitude of factors, such as advances in theory, the continuous technological progress in laser pulse power and intensity, more reliable numerical simulations and availability of ever increasing computational resources for these. The final goal is fundamental research and applied research with society-relevant applications, therefore the need for facility grade, laser driven particle accelerators that are cheaper, 
much more compact, and sometime more suited to the purpose than their "traditional" counterparts.

\subsection{Acceleration of electrons}

The acceleration of electrons is in a rapid progress, experiments proving higher electron energies with similar laser pulse powers. Recently an experiment at the BELLA laser in Berkeley ( [9]) reported 4.2 GeV electron bunches of $6 \mathrm{pC}$ with $6 \%$ energy spread, very well collimated ( $0.3 \mathrm{mrad}$ RMS divergence).

Laser Wake Field Acceleration (LWFA) in a gas-filled capillary discharge waveguide is the main technique applied for attaining the highest energies. Energies up to $40 \mathrm{GeV}$ are expected from simulations [13] using the parameter of ELI-NP laser and capillaries up to $50 \mathrm{~cm}$. Focusing parabola with focal length from $10 \mathrm{~m}$ up to $40 \mathrm{~m}$, corresponding to $\mathrm{F} / 20$ and possibly upgraded to F/80 in full beam aperture, are considered in the implementation, together with adequate electron magnetic spectrometers and beam dump.

\subsection{Acceleration of protons and heavy ions}

The laser-based acceleration of protons and heavy ions has the potential of producing beams with some major advantages with respect to classical accelerators when it comes to medical applications, such as the much lower secondary (prompt) ionizing radiation and activation of facility components.

Various ion acceleration mechanisms will be studied at ELI-NP in the new regimes of laser focus intensities approaching and hopefully even going beyond $10^{23} \mathrm{~W} / \mathrm{cm}^{2}$. Proton energies up to $500 \mathrm{MeV}$ are expected and taken as term source in beam dump and shielding calculations. However some of the physics cases require rather high fluxes and bunch densities at moderate energies, in the range of tens of $\mathrm{MeV} /$ nucleon for heavy ions.

The Radiation Pressure Acceleration (RPA) [8] mechanism is of highest interesting since it promises acceleration at solid state density which is many orders of magnitude above the density of ion bunches produced by any classical acceleration and opens therefore perspective for new studies. Other acceleration mechanisms such as Break Out Afterburner acceleration (BOA) [7] and Collisionless Shock Acceleration (CSA) in near critical density gas targets are proposed to be studied. CSA has been demonstrated experimentally using $\mathrm{CO}_{2}$ lasers $[5,14]$ and according to simulations, should enhance the efficiency of laser acceleration of protons and ions compared to TNSA (Target Normal Sheath Acceleration) as well as ion energy scaling [6]. 
Acceleration of heavy ions from solid targets is the only one giving the access to full range of elements and nuclear reactions of interest. The methods for energy selection and angular spread reduction (refocusing) are relevant for many applications and also proposed for study at ELI-NP.

\section{ELI-NP experiments using accelerated particles}

\subsection{Experimental areas and expected accelerated particles}

Out of the eight experimental areas at ELI-NP, four are devoted to laserbased experiments at the three power levels available, and one is devoted to experiments with combined laser and gamma/electron beams.

The E1 and E6 experimental areas feature both 10PW beams. In E1 it is foreseen the acceleration by RPA of protons and heavy ions, up to $500 \mathrm{MeV}$ (protons) or $50 \mathrm{MeV} / \mathrm{A}$ (heavy ions), and their use in laser-driven Nuclear Physics experiments. Accelerated proton bunches should have more than $10^{10} \mathrm{p} /$ pulse, energy FWHM of $10 \%$ and divergence better than $5^{\circ}$. E6 [15] is dedicated to fundamental physics research in High Field Physics and QED Experiments, aiming the study of Radiation Reaction and High Field Quantum Electrodynamics mainly through the Non-linear Compton Scattering and Multiphoton Breit-Wheeler Process. The experiments will use for the first time two such powerful laser beams in a pump-probe configuration: first 10PW laser beam (pump) focused on a gas or solid target accelerating electrons to multi-GeV, relativistic, energies; the second $10 \mathrm{PW}$ laser beam (probe) is tightly focused on the relativistic electron bunch generating the QED processes [10].

The E7 experimental area is devoted to experiments in fundamental physics with combined laser and gamma or electron beams. The laseraccelerated electron beams envisaged to be used in radiation reaction, pair creation and vacuum birefringence experiements will have energies up to 5 $\mathrm{GeV}$ and low divergence (less than $\sim 0.3 \mathrm{deg}$ ) and energy spread. These beams will be used for the generation of gamma photons in the GeV range by Compton backscattering effect.

The experiments at E4 and E5 employing 0.1 and 1PW laser beams at higher repetition rates are covering a wide range of topics, from materials science to the quest for dark matter candidates. Protons up to $60 \mathrm{MeV}$ in large bunches of $\sim 10^{10}-10^{12}$, and electrons in the range of $50-2,000$ $\mathrm{MeV}$ and intensities of up to a few $10^{10} /$ pulse are foreseen to be produced. Thermal electrons will also be generated at intensities of $\sim 10^{7} /$ pulse and moderate divergence of a few degrees. In E5 the study of materials behaviour 
in extreme environments will be a central topic, with a direct application to the development of accelerator components and societal applications like the understanding of structural materials degradation in next generation fusion and fission reactors or the shielding of equipment and human missions in outer space.

\subsection{Research proposals}

More than 100 researchers contributed to the ELI-NP Whitebook [3] proposing the first ideas for the experimental program at the new facility. The next step was detailing the categories of experiments in the TDRs. From the multitude of proposals employing the laser acceleration of particles few are presented below, in their general lines.

\section{Enhanced decay of 26Al in hot plasma environments}

The ${ }^{26} \mathrm{Al}$ nucleus was the first radioisotope detected in the interstellar medium, by the observation of the characteristic $1809 \mathrm{keV} \gamma$-emission associated with the decay of its ground state [12]. There is still a debate regarding the production sites of this isotope, that is a marker of ongoing nucleosynthesis. Moreover, in stellar conditions (temperatures up to $\sim 0.4 \mathrm{GK}$ ) it is predicted through theoretical calculations [2] that the effective life time of ${ }^{26} \mathrm{Al}$ would be reduced by many orders of magnitude due to a variety of physical processes.

At ELI-NP there is the possibility to produce plasma conditions similar to the ones in various astrophysical setups, and thus the population of the states of interest will provide a disintegration rate closer to the true astrophysical one [17]. The fact that the radiation pulses are ultrashort in time and synchronous will provide good conditions for the resolution of ps time scales.

\section{Production of extremely neutron-rich isotopes}

The waiting point around $\mathrm{N}=126$ is extremely important for the $r$ process nucleosynthesis, and presently very little is known about the nuclei in this area. The proposal [4] aims producing these nuclei by fissioning a dense thorium ion bunch with about $7 \mathrm{MeV} / \mathrm{u}$ in a thick thorium target (covered by a thin carbon layer), where the light fission fragments of the beam fuse with the light fission fragments of the target. The accelerated ${ }^{232} \mathrm{Th}$ bunches of solid state density are produced via the RPA process with a high intensity laser pulse (in the range of $10^{23} \mathrm{~W} / \mathrm{cm}^{2}$ ) produced by one of the 10PW HPLS arms. These bunches pass through a thin carbon layer and desintegrate into light and heavy fission fragments. In addition light ions $(\mathrm{H}, \mathrm{C})$ from the $\mathrm{CH} 2$ backing of the first (thin) Th target will be accelerated 
as well, inducing the fission process of ${ }^{232} \mathrm{Th}$ in the second, thick Th foil.

\section{Electron screening in astrophysical plasmas}

The measurement of rates and cross-sections (S-factors) in conditions close to the astrophysical ones (extremely low energies and including plasma effects) in the laboratory has been a great challenge for the experimental nuclear physics for many decades. A setup is proposed based on two high power laser beams generating, by interaction with a solid and a gas target, two colliding plasma bunches. The study of the role played by free and bounded electrons on the coulombian screening is one of the first topics to be investigated, but also other subjects are proposed. The TNSA acceleration regime demonstrated in previous experiments [11] will be acting for the solid thin target, while the other target will be a gas jet. Two regimes for the secondary (gas) target are envisaged.

\section{RA5 - electron acceleration}

At Research Activity 5 at ELI-NP, experiments with combined laser and gamma / electron beams are envisaged. Laser acceleration of electrons is needed for the proposed experiments in two energy ranges, one up to a few tens of $\mathrm{MeV}$ and one of $2.5-5 \mathrm{GeV}$. In the first case, a high intensity laser pulse will generate dense accelerated bunches of electrons that will be used for Bremsstrahlung radiation, which will interact with a secondary target and promote some isotopes of interest to isomeric states. In the second case, electrons are accelerated to $2.5-5 \mathrm{GeV}$ by one $1 \mathrm{PW}$ laser pulse, with the aim to produce high energy (up to $\mathrm{GeV}$ level) secondary gama beams after their interaction with another laser pulse. Studies of radiation reaction, gamma polarization and vacuum birefringence are proposed.

\section{Conclusions}

The acceleration of particles, electrons and protons/heavy ions, is a research and development area in extremely fast evolution. The possibility to obtain extremely high acceleration gradients and consequently accelerators of much reduced dimensions and costs is for sure a powerful driver for a very active research community. ELI-NP will focus on the study and perfectioning of these methods of acceleration, and on fundamental science employing the accelerated particles. Apart from laser science, important results in nuclear physics, astrophysics, life sciences, materials science and fundamental physics are expected from the new research infrastructure. 


\section{Acknowledgments}

The authors acknowledge the support from the Extreme Light Infrastructure Nuclear Physics (ELI-NP) Phase I, a project co-financed by the Romanian Government and European Union through the European Regional Development Fund.

\section{References}

[1] Cheriaux G., Giambruno F., Freneaux A., Leconte F. et al, AIP Conference Proceedings on Light at Extreme Intensities, 1462 (2012) 78

[2] Coc A. et al., Phys. Rev. C, 61 (1999) 015801

[3] ELI-NP White Book, http://www.eli-np.ro/documents/ELI-NPWhiteBook.pdf

[4] Habs D. et al., Applied Phys. B 103 (2011) 485

[5] Harberger D. et al., Nature Physics 8 (2012) 95

[6] d'Humières E. et al., Phys. of Plasmas 20 (2013) 023103

[7] Jung D. et al., New Journal of Physics 15 (2013) 023007

[8] Kar S. et al., Phys. Rev. Lett. 109 (2012) 185006

[9] Leemans W.P. et al, Phys. Rev. Letters, 113 (2014) 245002

[10] Luo W. et al., Phys. of Plasmas 22 (2015) 063112

[11] Macchi A. et al., Rev of Mod. Phys. 85 (2013) 751

[12] Mahoney W.A. et al., Astrophysical Journal, 286 (1984) 578

[13] Martins S.F., Fonseca R. A., Lu W., Mori W. B., Silva L. O., Nature Physics 6 (2010) 311

[14] Palmer C. et al., Phys. Rev. Lett. 106 (2011) 014801

[15] Turcu I.C.E., Balascuta S., Negoita F., Jaroszynski D., McKenna P., Proc. CSSP14, AIP Conf. Procs. 1645 (2015) 416

[16] Ursescu D.,Tesileanu O., Cernaianu M., Gales S., Zamfir N.V., Review of Laser Engineering, 42 (2014) 123

[17] Utsunomiya H. et al., Nucl. Phys. A 777 (2006) 459 